\title{
Many-body approach to the terahertz response of Wigner molecules in gated nanowire structures
}

\author{
K. M. Indlekofer, ${ }^{1,2, *}$ R. Németh,,${ }^{1,3}$ and J. Knoch ${ }^{4}$ \\ ${ }^{1}$ Center of Nanoelectronic Systems for Information Technology (CNI), IBN-1, Research Center Jülich GmbH, \\ D-52425 Jülich, Germany \\ ${ }^{2}$ FH Wiesbaden University of Applied Sciences, Information Technology and Electrical Engineering, Am Brückweg 26, \\ D-65428 Rüsselsheim, Germany \\ ${ }^{3}$ Institute of Electrical Engineering, Centre of Excellence CENG, Slovak Academy of Sciences, Dúbravská cesta 9 , \\ SK-841 04, Bratislava, Slovakia \\ ${ }^{4}$ IBM Research GmbH Zurich Research Laboratory, 8803 Rueschlikon, Switzerland \\ (Received 6 July 2007; revised manuscript received 13 December 2007; published 28 March 2008)
}

\begin{abstract}
In this article, we describe a numerical many-body approach for the description of the electromagnetic response of discrete one-dimensional electronic nanosystems. This approach is based on a recursive method to construct a subset of relevant Slater determinants as a many-body basis for the considered system. In turn, we employ a generalized Floquet theory to calculate the periodic many-body statistical operator for the system which is subject to a periodic time-dependent Hamiltonian. As an application of this method, we propose a $\mathrm{THz}$ probe technique to obtain spatially resolved information about the electronic spectra inside gated nanowires. This spectroscopic approach employs a segmented multigate design for the local detection of quantum transitions between few-electron states. The obtained simulation results for the intraband $\mathrm{THz}$ response spectrum show fingerprints for the formation of Wigner molecules inside the nanowire in the long channel limit.
\end{abstract}

DOI: 10.1103/PhysRevB.77.125436

PACS number(s): 73.43.Cd, 73.21.Hb, 73.50.Mx

\section{INTRODUCTION}

The electronic properties of ultimately scaled nanostructures are dominated by only a handful of electrons or holes. In such nanodevices, one has to face two nonclassical physical mechanisms. ${ }^{1,2}$ First, due to the spatial confinement of charge carriers, quantization energies of electronic states become relevant, leading to a nonclassical transport behavior. Second, the details of the Coulomb interaction between the individual carriers become important and can no longer be described in terms of a mean-field picture. As a prototype system, one-dimensional nanowire-based structures ${ }^{3-5}$ have recently attracted great interest due to their advantageous electrostatics and transport properties. ${ }^{6-8}$ From a different perspective, they also represent model systems for the study of technological as well as physical challenges in future nanodevice designs.

In order to capture the numerous details of a realistic nanostructure, such as the actual geometrical configuration of the gate electrodes, a realistic quantum simulation of such a system requires the consideration of a sufficiently large number $Z \approx 50-500$ of single-particle states. ${ }^{9,10}$ Normally, due to the lack of symmetries, such systems are inhomogeneous and anisotropic and furthermore may exhibit a longrange interaction. Since the resulting many-body problem scales exponentially in $Z$, a full diagonalization thus grows beyond any computational limit. As an option, one could take the path to consider a highly idealized model (analogous to the Anderson or Hubbard models ${ }^{11,12}$ ) mapping the actual nanosystem to a reduced (albeit nontrivial) model system with a small number of effective parameters. Such an approach can be justified in the sense that typically only a small number of degrees of freedom are relevant for the investigated fundamental effects. Nevertheless, these relevant degrees of freedom and their associated effective parameters in general are nonlinear functions of the actual experimentally accessible parameters (such as gate voltages) and not known a priori. In order to address this problem, we have recently introduced a multiconfigurational approach ${ }^{8,13,14}$ (MCSCG) which employs a reduced adaptive basis for the simulation of stationary Coulomb blockade effects in gated nanowire structures.

For the realistic simulation of nonlinear and nonadiabatic real-time response phenomena in such finite (or quasiisolated) many-body nanosystems, a large number of excited many-body eigenstates has to be considered. Here, manybody approaches that focus on ground-state or equilibrium properties of the system, such as density functional theory ${ }^{15-17}$ (DFT) or the quantum Monte Carlo ${ }^{18-20}(\mathrm{QMC})$ method are not suitable. For the considered problem, the configuration interaction (CI) method $^{21-28}$ is the most flexible approach, based on an expansion of the many-body eigenstates in terms of simple many-body basis functions such as Slater determinants. However, due to the exponentially growing number of states, standard CI implementations are typically restricted to a small number of single-particle basis states or limited excitation degrees [e.g., single-double (SD-) CI and multireference-double-excitation (MRD-) $\mathrm{CI}] .{ }^{23,25,26,29}$ As a generalization, the Monte Carlo CI (MCCI) has been introduced in Refs. 30 and 31, using an iterative optimization procedure by repeated Monte Carlo based configuration generation, diagonalization, and selection of the most important configurations. However, if a large number of excited many-body eigenstates has to be computed as in our problem, the MCCI might not be computationally efficient enough due to the need for a repeated diagonalization.

In order to address these issues of a realistic many-body simulation, in this paper we therefore introduce a recursive scheme which is able to generate a priori a large number of configurations (Slater determinants, typically $10^{3}-10^{6}$ ) without the need for a costly diagonalization within the selection 
process. The algorithm consists of two steps: (i) recursive construction of a large set of relevant Slater determinants ("bucket-brigade algorithm") and (ii) many-body diagonalization within the resulting Fock subspace. Such an approach enables us to describe correlated many-body states beyond mean-field theory which become essential within the field of quantum information technology.

In the following sections, we first describe the main physical ideas behind our many-body simulation approach. Subsequently, we demonstrate the strengths of the concept by considering a realistic example of a gated nanowire device. From the simulated THz intraband response of this system, we observe indications of the formation of a Wigner molecule in the long channel limit, yielding significant deviations from the single-particle picture.

\section{RELEVANT MANY-BODY SUBSPACE}

For an arbitrarily given single-particle orthonormal (ON) basis $B=\left\{\phi_{j}\right\}$ the exact many-body Hamiltonian of the finite (i.e., discrete) system in second quantization reads as

$$
H_{0}=\sum_{j, k} \epsilon_{j k} c_{j}^{\dagger} c_{k}+\frac{1}{2} \sum_{j, k, l, m} V_{j k l m} c_{j}^{\dagger} c_{k}^{\dagger} c_{l} c_{m},
$$

where $\epsilon_{j k}$ and $V_{j k l m}$ denote single-particle and Coulomb twoparticle matrix elements of the total system Hamiltonian and $c_{j}$ is the annihilation operator for the single-particle state $j$.

For a numerical diagonalization of this many-body Hamiltonian, we employ Slater determinants as simple many-body basis states, constructed from the single-particle basis $B$. Obviously, for numerical reasons, one has to select a finite subset of relevant Slater determinants from the infinitely large set of all basis states. In the following, we describe a recursive algorithm for the deterministic generation of such a relevant subset which goes beyond the commonly employed truncation scheme based on $n$ th-order excitation. $^{25,26}$

\section{A. Bucket-brigade algorithm: Recursive construction of relevant Slater determinants}

Let $N_{\max }$ be the maximum number of single-particle states that shall be considered. Hence, we consider a subspace spanned by $B_{N_{\max }}=\left\{\phi_{j} \mid j=0, \ldots, N_{\max }-1\right\}$. The recursion procedure can be formulated in terms of a sequence of sets $S_{J, N}$ of Slater determinants. Here, $J=0, \ldots, N_{\max }$ denotes the recursion step number and $N=0, \ldots, N_{\max }$ is the particle number. Each $S_{J, N}$ can be interpreted as a "bucket," containing relevant Slater determinants of $N$ particles in single-particle states $j=0, \ldots, J-1$. Within each recursion step $J \rightarrow J+1$, we first expand the old sets $S_{J, N}$ by adding states of $S_{J, N-1}$ with an extra particle created in the single-particle state $j=J$. After this expansion, the buckets are truncated again by selecting only the most suitable Slater determinants. For this purpose, we employ a suitable "measure of importance" $\mu$ for the selection of Slater determinants within each bucket. The described procedure is visualized in Fig. 1. In the Appendix, all technical aspects of the formal recursion,

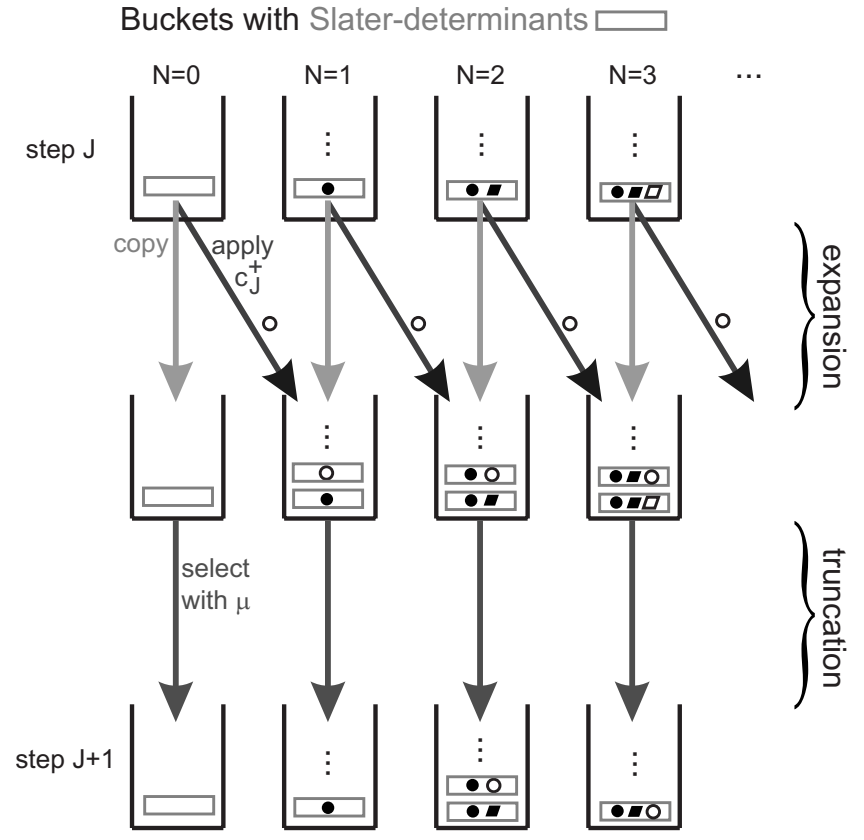

FIG. 1. Visualization of the recursion step $J \rightarrow J+1$ : for a considered bucket $N$ copy all existing states, add new states by applying $c_{J}^{\dagger}$ to all existing states from the previous bucket $(N-1)$ and truncate with the help of the measure $\mu$. A small circle or rhombus represents a single-particle state, and $N$ denotes the particle number in the bucket. (The shown truncation is an artificial example.)

1. Start: Empty buckets (except for the bucket containing the vacuum state)

2. Recursion step $J \rightarrow J+1$ : Add single-particle state $J$ to existing Slater determinants and select the most relevant ones by use of $\mu$

and the choice of $\mu$, are discussed in detail.

The main benefit of this "bucket-brigade CI" (BBCI) recursion stems from a significant reduction of the number of Slater determinants $D_{N} \ll D_{\max }(N)$ that have to be considered numerically. Here,

$$
D_{\max }(N)=\left(\begin{array}{c}
N_{\max } \\
N
\end{array}\right)
$$

denotes the maximum number of Slater determinants for $N$ particles. In turn, the obtained "relevant" subset of Slater determinants is employed as a many-body basis for a subsequent diagonalization of the many-body Hamiltonian within the relevant subspace. Finally, with the help of the resulting relevant eigenvectors and associated eigenvalues of the many-body Hamiltonian one can calculate the time evolution of the isolated system and can construct stationary statistical operators for the calculation of expectation values.

\section{B. Approximations and applicability}

By considering only those Slater determinants that are chosen as "relevant" by the described algorithm, two controlled approximations are made: First, the number of considered single-particle states $N_{\max }$ is finite. Equivalently, the 
recursion step is performed only a finite number of times $N_{\max }$. Second, the maximum number $D_{N}$ of Slater determinants in each set $S_{J, N}$ can be smaller than $D_{\max }(N)$. In the limit $N_{\max } \rightarrow \infty$ and $D_{N} \rightarrow \infty$, the algorithm becomes exact.

The described algorithm typically becomes advantageous if the required maximum number of single-particle states $N_{\max }$ and the number of particles $N$ provide a maximum number $D_{\max }(N)$ of Slater determinants that lies beyond the limit for which the resulting problem can be fully diagonalized (full CI), in particular for realistic nanoelectronic systems which are strongly inhomogeneous and anisotropic with minimal symmetries. Consequently, the single- and twoparticle matrix elements of the Hamiltonian have to be considered as arbitrarily given quantities, lacking the symmetries of idealized impurity and lattice models for which renormalization group (RG) approaches ${ }^{32,33}$ would become advantageous. For the considered nanosystem, typical application-relevant values are $N_{\max }=32-256, N=0-32$, and $D_{N}=10^{3}-10^{6}$. For such a problem, RG-based approaches might become impractical since the storage requirements of matrices for the given $N_{\max }$ and $D_{N}$ grow beyond realistic limits.

In principle, the described recursive algorithm is suitable to provide a basis for many-body states up to any energy scale since all single-particle states are scanned systematically. It can be used in particular for nanoelectronic manybody problems where a single set of Slater determinants has to be determined which is relevant for a whole energy interval of many-body eigenstates (not only the ground state or a particular excited state). As a major advantage, the BBCI approach is able to calculate a large set of excited manybody states, which are mandatory for the simulation of nonlinear real-time response properties. In principle, a full-CI approach would also provide such information, however, at the cost of an exponentially increased number of many-body basis states. For example, a number $N_{\max }=64$ single-particle states for $N=4$ electrons would imply a total number of $D_{\text {max }}(N)=635376$ Slater determinants, whereas the BBCI typically selects only a few 1000 final relevant Slater determinants (see example in Sec. IV). Consequently, a significant reduction of total computational time for the subsequent numerical diagonalization is obtained. From a different perspective, the BBCI algorithm constitutes a generalization of the SD-CI and MRD-CI techniques by use of a selection measure $\mu$. In order to demonstrate the strengths of the BBCI quantitatively, a numerical comparison of the BBCI with a conventional CI-based approach for the case of a gated nanowire system will be provided in Sec. IV C.

In general, CI-based approaches become beneficial for systems with a relatively small number of single-particle basis states which are involved in correlation (or subject to occupation fluctuations). Here, zero- and one-dimensional (OD and 1D) nanoelectronic systems represent suitable application-relevant candidates, as discussed in this paper.

By construction, the BBCI generates $N$-particle manybody basis states by recursively expanding those of $N-1$ particles. Such an approach assumes that those interaction effects that are relevant for $N-1$ particles are also important for the $N$-particle system. In order to address this point, the measure $\mu$ in principle can be constructed such that the "missing" electron in $N-1$ can be included in terms of an $a$ posteriori mean-field contribution in $\mu$. Furthermore, it is important to keep track of a large number (typically $10^{4}-10^{7}$ ) of states within the intermediate buckets to prevent from removal of states which eventually might become important for the final bucket. Such a constraint, however, can be easily fulfilled numerically since the BBCI recursion scheme does not require any intermediate diagonalization steps but solely the storage and manipulation of a corresponding number of integers (such as the bitmap representations of Slater determinants). Since the BBCI algorithm generates states with particle numbers up to a given $N_{\max }$, the latter states can be employed as a basis for grand canonical as well as canonical calculations.

In conclusion, the BBCI technique offers the advantage of a significantly reduced set of many-body basis states in combination with the ability to calculate a large set of excited many-body eigenstates. In turn, expectation values of arbitrary subspace observables with respect to any given statistical operator can be calculated numerically (not necessarily restricted to the ground state or equilibrium). A typical example of a realistic nanosystem where these advantages can be exploited will be discussed in Sec. IV, considering the $\mathrm{THz}$ response of a gated 1D nanowire system. The full potential of the BBCI remains to be explored yet.

\section{PERIODIC STATISTICAL OPERATOR}

The Hamiltonian $H(t)$ of the system is assumed to be periodic

$$
H(t+T)=H(t) .
$$

Hence, we can write

$$
H(t)=\sum_{m=-\infty}^{+\infty} e^{-i m \omega t} H_{m},
$$

with "Fourier coefficient" operators $H_{m}$ and $\omega \equiv 2 \pi / T .34,35$ Note that the oscillatory terms $H_{m \neq 0}$ are not necessarily small perturbations of $H_{0}$. In the following, we consider the general case, not assuming any particular form of $H_{m \neq 0}$.

The many-body statistical operator $\rho$ of the system obeys a generalized Liouville equation

$$
\frac{\partial \rho(t)}{\partial t}=-\frac{i}{\hbar}[H(t), \rho(t)]-\frac{\epsilon}{\hbar}[\rho(t)-\tilde{\rho}],
$$

where $\tilde{\rho}$ is a given statistical operator (such as thermodynamical equilibrium for $H_{0}$ or electronically driven nonequilibrium) with an empirical relaxation constant $\epsilon \rightarrow 0+$. In the following, we are interested in periodic solutions of this equation of motion, corresponding to a "stationary" limit cycle of the system. Hence, we can write

$$
\rho(t)=\sum_{m=-\infty}^{+\infty} e^{-i m \omega t} \rho_{m}
$$

with "Fourier coefficient" operators $\rho_{m}$. Employing the equation of motion, we thus obtain 


$$
m \hbar \omega \rho_{m}-\sum_{m^{\prime}=-\infty}^{+\infty}\left[H_{m-m^{\prime}}, \rho_{m^{\prime}}\right]=i \epsilon\left(\delta_{m, 0} \tilde{\rho}-\rho_{m}\right)
$$

which is equivalent to Floquet theory $29,34,35$ in Liouville space if $\epsilon \equiv 0$.

\section{A. Projection operator}

In order to formulate the exact solution for the operator set $\left\{\rho_{m}\right\}$ in a compact form, we make use of a "Floquet superoperator" notation: Choosing an arbitrary but fixed ON basis of the considered Fock space, we can rewrite Eq. (7) in this representation as

$$
\begin{aligned}
& m \hbar \omega \rho_{m, j, k}-\sum_{m^{\prime}, j^{\prime}} H_{\left(m-m^{\prime}\right), j, j^{\prime}} \rho_{m^{\prime}, j^{\prime}, k}+\sum_{m^{\prime}, k^{\prime}} \rho_{m^{\prime}, j, k^{\prime}} H_{\left(m-m^{\prime}\right), k^{\prime}, k} \\
& \quad=i \epsilon\left(\delta_{m, 0} \widetilde{\rho}_{j, k}-\rho_{m, j, k}\right),
\end{aligned}
$$

where $j, j^{\prime}, k, k^{\prime}$ denote Fock space basis state indices. Employing multi-indices $J=(m, j, k), J^{\prime}=\left(m^{\prime}, j^{\prime}, k^{\prime}\right)$ we finally get

$$
\sum_{J^{\prime}} L_{J, J^{\prime}} v_{J^{\prime}}+i \epsilon v_{J}=i \epsilon \widetilde{U}_{J}
$$

with the "Floquet supermatrix"

$$
L_{J, J^{\prime}}=m \hbar \omega \delta_{m, m^{\prime}} \delta_{j, j^{\prime}} \delta_{k, k^{\prime}}-H_{\left(m-m^{\prime}\right), j, j^{\prime}} \delta_{k, k^{\prime}}+\delta_{j, j^{\prime}} H_{\left(m-m^{\prime}\right), k^{\prime}, k},
$$

and "Floquet supervectors" which are defined via

$$
v_{J}=\rho_{m, j, k} \quad \text { and } \quad \widetilde{v}_{J}=\delta_{m, 0} \widetilde{\rho}_{j, k},
$$

providing a one-to-one correspondence between Floquet supervectors $v$ and operator sets $\left\{\rho_{m}\right\}$. In a more compact form, this can be written as

$$
L v+i \epsilon v=i \epsilon \widetilde{v} .
$$

Since $H(t)$ is Hermitian, the Floquet supermatrix $L$ becomes Hermitian as well. Hence, $L$ exhibits real eigenvalues only and $L+i \epsilon$ is a regular matrix. In turn, the equation for the Floquet super-vector $v$ can be solved uniquely:

$$
v=\frac{i \epsilon}{L+i \epsilon} \widetilde{v}
$$

As can be readily shown, in the limit $\epsilon \rightarrow 0+$, we get

$$
\lim _{\epsilon \rightarrow 0+} \frac{i \epsilon}{L+i \epsilon}=P_{0},
$$

where $P_{0}$ is the projection operator (in Floquet superspace) to the eigenspace of $L$ for eigenvalue 0 . Hence, in this limit, we get

$$
v=P_{0} \tilde{v} \quad(\epsilon \rightarrow 0+) .
$$

One has to note that $L$ depends on $\omega$. Therefore, $P_{0}$ becomes $\omega$ dependent as well. Furthermore, $P_{0}$ involves all orders in $\left\{H_{m}\right\}$, which makes this approach nonperturbative. In contrast to the case $\epsilon \equiv 0$ which yields no unique solution $v$ in general, the upper expression provides a unique solution, derived in a continuous manner from the initial preparation $\widetilde{v}[\tilde{\rho}]$. As can be shown, the resulting $\rho(t)$ is

(i) $T$ periodic,

(ii) Hermitian,

(iii) positive semidefinite (i.e., all eigenvalues $\geq 0$ ),

(iv) and obeys $\operatorname{Tr}[\rho(t)]=1$,

provided that $\widetilde{\rho}$ is a valid statistical operator. Note that, in general, we obtain $\rho_{m} \neq \delta_{m, 0} \widetilde{\rho}$ (except for trivial cases such as $\left[H_{m}, H_{m^{\prime}}\right]=0$ with $\left.\left[H_{m}, \tilde{\rho}\right]=0\right)$.

For practical reasons, the maximum harmonic index $m$ must be truncated at some sufficiently large number $m_{\max }$. One has to note that such a truncation in harmonic index $m$ up to $m_{\max }$ differs significantly from Kubo perturbation theory ${ }^{36,37}$ up to $m_{\max }$-th order in $h \equiv H-H_{0}$. (i) The projection operator approach in principle accounts for all orders in $h$ that contribute to a given harmonic index $m$, albeit involving the approximation of a finite $m_{\max }$. (ii) In contrast, perturbation theory considers only a given finite order in $h$, and thus neglects all possible contributions of higher orders in $h$ to a given harmonic $m$. As such, the projection operator provides a nonperturbative approach to the time-dependent problem $H(t)$, beyond standard Kubo theory.

For a numerical implementation, we assume that the eigenstates and eigenenergies of $H_{0}$ are known within a sufficiently large relevant subspace, calculated via the bucketbrigade algorithm as described in the previous section. In turn, all operators are written as matrices with respect to this eigenbasis within the relevant subspace of dimension $n_{\max }$ $\leq D_{N_{0}}$. Within the studied realistic examples, it is sufficient to employ $n_{\max } \approx 100$ many-body basis states (of $D_{N_{0}}$ $\approx 10^{3}-10^{4}$ ) with up to $m_{\max } \approx 10$ harmonics. Since

$$
\rho_{-m}=\rho_{m}^{\dagger}, \quad H_{-m}=H_{m}^{\dagger},
$$

it is sufficient to keep track of matrices with $m \geq 0$. Consequently, we have to deal with Floquet supervectors $v$ with $d=\left(m_{\max }+1\right) n_{\max }^{2} \approx 10^{5}$ complex elements, yielding Floquet supermatrices of the size $d \times d$. (Note that conventional Liouville supermatrices have the size $d^{\prime} \times d^{\prime}$ with $d^{\prime}=n_{\max }^{2}$.) Here, a significant reduction of computational requirements arises for the application-relevant case of a monochromatic perturbation, where $H_{m} \neq 0$ only for $m=0, \pm 1$, which provides sparse matrices $L$.

\section{B. Density-matrix recursion}

For small perturbation strengths, a computationally more efficient technique can be formulated: With the notation $[A, \cdot] \rho_{m}:=\left[A, \rho_{m}\right]$, we can rewrite Eq. (7) as

$$
\left(m \hbar \omega-\left[H_{0}, \cdot\right]+i \epsilon\right) \rho_{m}=\sum_{m^{\prime} \neq m}\left[H_{m-m^{\prime}}, \rho_{m^{\prime}}\right]+i \epsilon \delta_{m, 0} \tilde{\rho} .
$$

With the unperturbed resolvent Liouville superoperator

$$
R_{m}=\left(m \hbar \omega-\left[H_{0}, \cdot\right]+i \epsilon\right)^{-1}
$$

we therefore obtain the "density-matrix recursion" formula 


$$
\rho_{m}^{(M+1)}=(1-\alpha) \rho_{m}^{(M)}+\alpha R_{m}\left(\sum_{m^{\prime} \neq m}\left[H_{m-m^{\prime}}, \rho_{m^{\prime}}^{(M)}\right]+i \epsilon \delta_{m, 0} \tilde{\rho}\right)
$$

for step $M \rightarrow M+1$, where $\alpha$ is a numerical damping parameter. As the "initial condition" for this recursion at step $M$ $=0$ we choose $\tilde{\rho}$ :

$$
\rho_{m}^{(0)}=\delta_{m, 0} \tilde{\rho} .
$$

This choice ensures a unique preparation of the system, being physically related to the preparation $\widetilde{\rho}$. Provided that this recursion schemes converges, its solution obviously fulfills the equation of motion. As a special case, a finite number of recursion steps with $\alpha=1$ stands in direct correspondence to time dependent perturbation theory. ${ }^{36,37}$

Within a numerical implementation, this recursion technique becomes advantageous in the eigenbasis of the unperturbed Hamiltonian $H_{0}$ since $R_{m}$ becomes diagonal as well. As for the domain of convergence, the density-matrix recursion proves useful for small perturbation strengths, providing identical results as compared to the projection operator technique.

\section{Expectation values and correlation functions}

Finally, we can calculate the time dependent expectation value for an arbitrarily given observable $A$ as

$$
\langle A\rangle(t) \equiv \operatorname{Tr}[A \rho(t)]=\sum_{m} e^{-i m \omega t} A_{m}
$$

with Fourier coefficients $A_{m}=\operatorname{Tr}\left(A \rho_{m}\right)$. Note that due to the $T$ periodicity of $\rho(t)$, any expectation value $\langle A\rangle(t)$ becomes $T$ periodic as well and hence exhibits a purely harmonic spectrum with fundamental frequency $\omega$ of the perturbation $h(t)$ $:=H(t)-H_{0}$. For example, the knowledge of $\langle d\rangle(t)$ with the dipole operator $d$ can be used for the calculation of stimulated (coherent) dipole emission with $h$ representing a periodic pump input signal.

In order to compute the $N$-point $(N \geq 2)$ correlation functions of operators $A_{n}$, we need to evaluate expectation values of the form

$$
\left\langle A_{1}\left(t_{1}\right) \cdots A_{N}\left(t_{N}\right)\right\rangle \equiv \operatorname{Tr}\left[A_{1}\left(t_{1}\right) \cdots A_{N}\left(t_{N}\right) \rho\right],
$$

where $A_{n}\left(t_{n}\right)$ are Heisenberg operators and $\rho \equiv \rho(t=0)$ is the statistical operator of the system. For example, an expression of the form $\left\langle d_{+}\left(t_{1}\right) d_{-}\left(t_{2}\right)\right\rangle$ is required for the calculation of the emission spectrum (coherent plus incoherent) according to a generalized Wiener-Khintchine theorem. ${ }^{38}$ We have $A(t)=U^{\dagger}(t) A U(t)$ with the propagator $U(t)$. Note that in contrast to $\rho(t+T)=\rho(t)$, we have $A(t+T) \neq A(t)$ in general. For $N \geq 2$, the knowledge of $\rho(t)$ is not sufficient and we need to know the explicit form of $U(t)$ as well. At least for $\rho$, we can employ the result

$$
\rho \equiv \rho(0)=\sum_{m} \rho_{m}
$$

As for the numerical calculation of

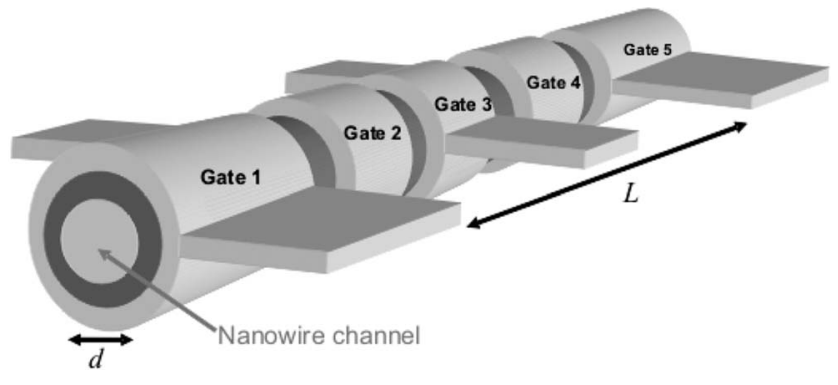

FIG. 2. Schematic view of an idealized nanowire in multigate configuration ("caterpillar-field-effect transistor"). This example shows a system with five coaxial gates. Here, gates 1 and 5 are negatively biased and thus provide barriers. Gates 2, 3, and 4 cover a channel region of length $L$ and act as probes. In the simulations, the intergate gaps are assumed to be negligible. The outer source and drain contacts are not shown here.

$$
U(t \geq 0) \equiv \mathrm{T} \exp \left(-\frac{i}{\hbar} \int_{0}^{t} d t^{\prime} H\left(t^{\prime}\right)\right)
$$

we can employ the periodicity of $H(t)$ and obtain

$$
U(t+T)=U(t) U(T) .
$$

Therefore, it is sufficient to integrate and store $U(t)$ for 0 $\leq t \leq T$ numerically (for a sufficiently small time step $\Delta t$ $\left.\sim 1 / \omega_{\max }\right)$ and employ $U(T)$ for the continuation to all times.

In summary, we have all the ingredients to calculate expectation values numerically for the given $H(t)$ with the help of the projection superoperator. Only correlation functions require the explicit evaluation of $U(t)$ for $0 \leq t \leq T$.

\section{EXAMPLE: WIGNER MOLECULES IN GATED NANOWIRES}

As an application of our method, we now consider the $\mathrm{THz}$ intraband response spectrum of a realistic gated nanowire system. Figure 2 shows a schematic sketch of the considered nanowire with multiple gate segments which serve as electrostatic probes on a nanoscale. In contrast to microwave-assisted tunneling 39,40 which is based on transport measurements, in our example we consider a quasiisolated system where oscillating image charges within the gate electrodes serve as a means to detect the motion of the confined electrons caused by the external $\mathrm{THz}$ field. We assume that only one lateral subband needs to be taken into account, corresponding to channel diameters in the sub30-nm range for GaAs. The following simulation results are based on the BBCI algorithm in combination with first-order density-matrix recursion [i.e., $\alpha=1$ and $M=0$ in Eq. (19)] for the description of the $\mathrm{THz}$ response spectra within the chosen relevant many-body basis. ${ }^{42}$ As for the numerical parameters of the following example, we have employed 400 tightbinding sites for the description of the nanowire channel. In turn, the BBCI algorithm was applied to the lowest 64 single-particle eigenstates of the single-particle Hamiltonian (including the spin degree of freedom), ordered by singleparticle energy. 
As for the numerical implementation, the selection measure $\mu$ as given in Eq. (A2) was employed. Finally, the number of relevant Slater determinants, which served as many-body basis states for the subsequent numerical diagonalization, was chosen to be 3072 in the final bucket (and up to 262144 states in the intermediate buckets).

For the following idealized example, the nanowire channel is covered by multiple separated gate electrodes as depicted in Fig. 2. Experimentally, such a structure could be realized by use of 1D semiconductor whiskers, ${ }^{3}$ conformally coated by the gate dielectric and metallic gate electrodes which are structured by means of electron-beam lithography. Applying a sufficiently large negative voltage to the outermost gates (for example, gates 1 and 5 in Fig. 2), barriers arise and we thus can assume an almost isolated channel system. A monochromatic input $\mathrm{THz}$ excitation is applied to gate 1, whereas the intermediate gate fingers (gates 2, 3, and 4 in Fig. 2) serve as spatially resolved probes. In the considered example, the outermost gates are biased to $-2 \mathrm{~V}$ and all the intermediate gates to $0 \mathrm{~V}$.

In order to illustrate the main effect, we consider two different nanowire field effect transistors with a GaAs channel and $\mathrm{SiO}_{2}$ gate insulation. In both cases we keep the channel diameter $d=20 \mathrm{~nm}$ and the oxide thickness $d_{\mathrm{ox}}=10 \mathrm{~nm}$ constant, whereas the channel lengths are chosen as $L=150$ and $600 \mathrm{~nm}$ with corresponding total oxide capacitances of $C_{\mathrm{ox}}=47 \mathrm{aF}$ and $C_{\mathrm{ox}}=188 \mathrm{aF}$, respectively. In the following example, $40+2$ gate segments will be considered. Due to the coaxial gate electrodes, the Coulomb interaction within the channel region is strongly screened. In the considered example, we obtain a screening length ${ }^{8,43}$ of $\lambda=10.5 \mathrm{~nm}$ for $\epsilon_{\mathrm{ox}}=3.9$ and $\epsilon_{\mathrm{ch}}=12.4$. In the following discussion, we assume a thermodynamical equilibrium state in the low temperature limit in order to obtain a well-resolved occupation of quantum states. The total electron number is kept fixed at $N=4$, trapped within the inner channel region $L$. As for a possible experimental preparation of such an electronic state, one could employ the outer source and drain contacts (not shown in Fig. 2) in combination with suitable barrier-gate voltages to make use of single-electron tunneling for electron counting. 44

\section{A. Charge density}

Figure 3 shows the simulated charge density along the channel axis for the nanowire with $L=150 \mathrm{~nm}$. For comparison, the noninteracting case is shown in Fig. 3(a), whereas the realistic case which includes the Coulomb repulsion is plotted in Fig. 3(b). For this case, the single-particle quantization energy dominates as compared to the Coulomb repulsion, resulting in a charge distribution which resembles the shape of a noninteracting system. Here, the Coulomb repulsion solely broadens the spatial electron distribution. In contrast, for the $L=600 \mathrm{~nm}$ case [Fig. 4(a) and 4(b)] one can clearly identify the formation of a charge-density wave, indicating the onset of the Wigner molecule regime ${ }^{45}$ with separated electrons owing to the dominating Coulomb repulsion. Comparing the artificial noninteracting case Fig. 4(a) with Fig. 4(b), one can clearly observe the qualitative change
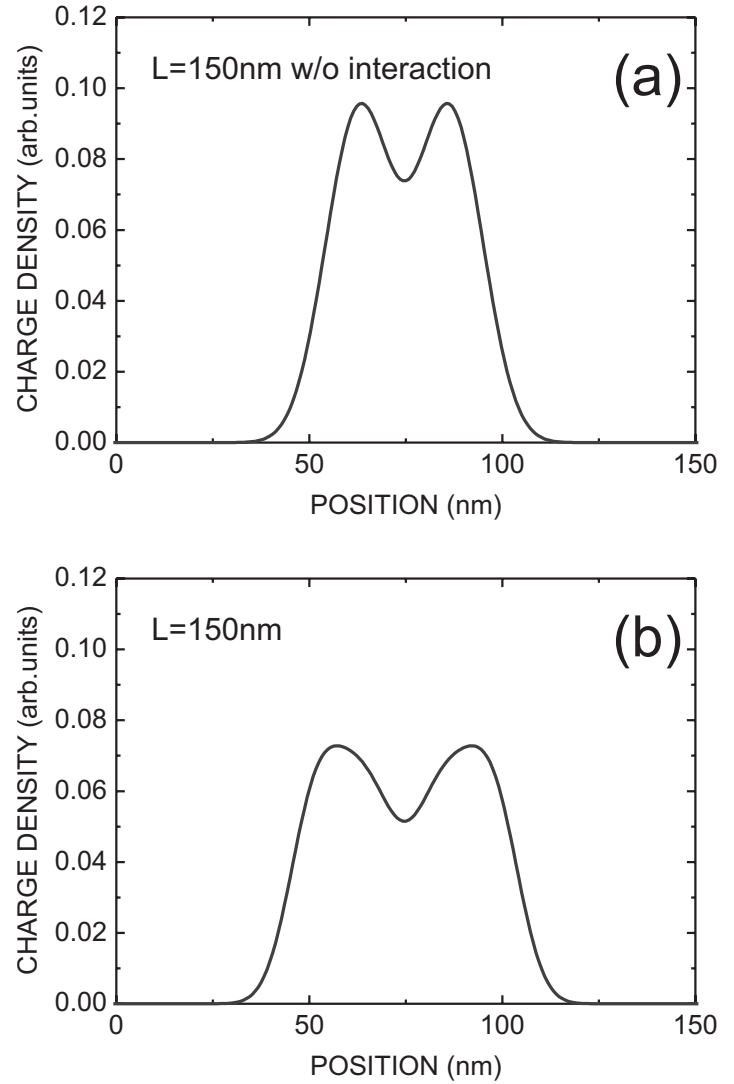

FIG. 3. Simulated electron charge density for a GaAs nanowire FET with $L=150 \mathrm{~nm}$ and $d=20 \mathrm{~nm}$. The number of electrons is $N=4$. (a) shows the noninteracting case, whereas (b) includes the electron-electron interaction. Comparing the two cases, no significant qualitative change can be observed.

in the electron-density profile, demonstrating the role of the Coulomb interaction in this case. The actual transition to the Wigner regime for a nanowire structure in general results from a competition between kinetic energy and Coulomb energy, which of course depends on the chosen materials and geometries. In the long-channel case $(L=600 \mathrm{~nm})$, the mean spatial separation of the electrons $\Delta x \simeq L / N$ becomes much larger than the screening length $\lambda$ of the Coulomb interaction within the channel. For the discussed example, in turn, it is energetically favorable for the electrons to form a chargedensity wave in order to reduce the Coulomb energy, outweighing the increased kinetic energy due to the spatial confinement $\Delta x$.

For a realistic nanodevice simulation in general, it is crucial to take the spin degree of freedom into account, even in the absence of an external magnetic field. (See, for example, Ref. 41. Here, charge-density waves have been observed as well.) In fact, within the BBCI scheme, the electronic spin is naturally included in terms of the chosen single-particle basis and associated matrix elements. One has to note that for our nanowire system without external magnetic field, we do not observe any broken spin symmetry since the BBCI manybody eigenstates correctly resemble the symmetry of the underlying Hamiltonian. In contrast, a single-Slaterdeterminant mean-field approximation might yield broken symmetries even in the absence of an external field. 

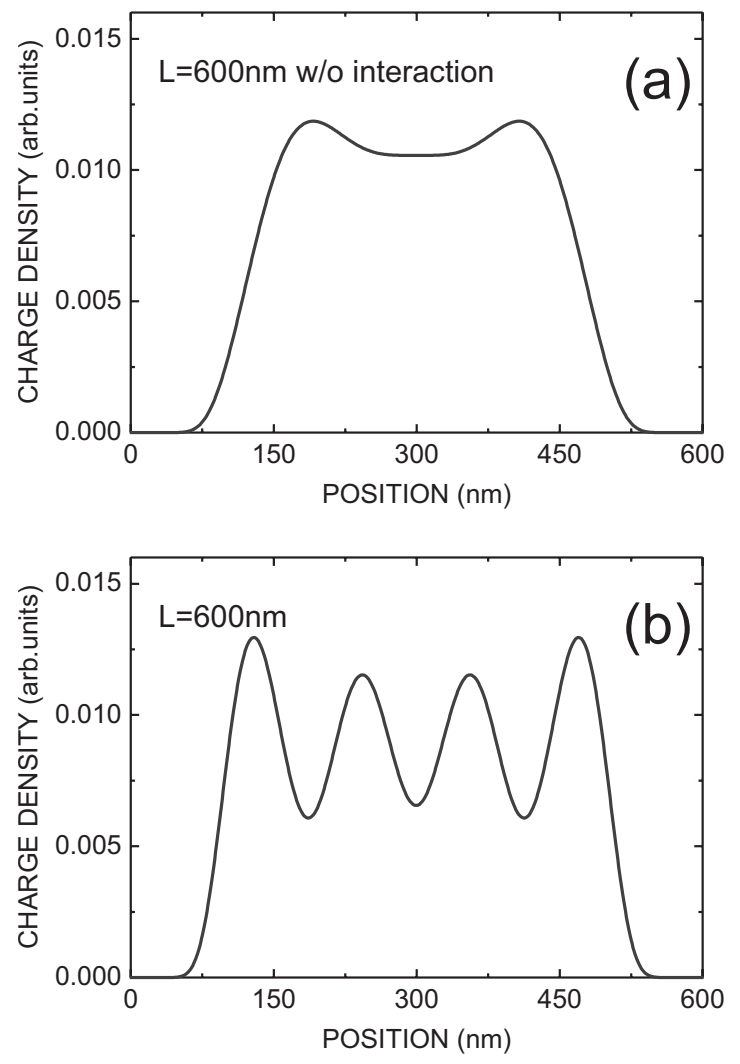

FIG. 4. Simulated electron charge density for a GaAs nanowire FET with $L=600 \mathrm{~nm}$ and $d=20 \mathrm{~nm}$. The number of electrons is $N=4$. (a) shows the noninteracting case, whereas (b) includes the electron-electron interaction. The charge-density wave in (b) indicates the formation of a Wigner molecule within the long-channel device. Here, the electron density differs qualitatively from the noninteracting case (a).

\section{B. Spatially resolved $\mathrm{THz}$ response}

In order to probe such a peculiar electronic configuration within a nanowire channel, we now consider the spatially resolved $\mathrm{THz}$ response spectrum of such a device. In the following simulation results, we have employed a resolution of 40 intermediate probe gates. Here, the physical observable that we consider for the output signal is nothing but the induced image charge within the individual gate electrodes. One has to note that each gate finger detects a spatially averaged signal within an effective interval $l_{G}^{\mathrm{eff}} \simeq l_{G}+2 \lambda$, where $l_{G}$ denotes the geometrical length of the gate segment and $\lambda$ the gate screening length. ${ }^{8}$ Parasitic stray capacitances which will likely occur in an experimental realization of such a device are not considered in this paper. For the experimental case it might be advantageous to employ alternating screening and signal gates, combined with an optimized $\mathrm{THz}$ layout and a suitable preamplification setup for the detection of the image charge signal. ${ }^{46}$

Figures 5 and 6 show the simulated first-order THz spectra for the two cases $L=150$ and $600 \mathrm{~nm}$, respectively, which exhibit fundamental resonances at $1.69 \mathrm{THz}$ and $70 \mathrm{GHz}$. The higher value for the $150 \mathrm{~nm}$ case stems from a narrower electron distribution [see Fig. 3(b)]. Most noticeably, comparing the qualitative form of the two spectra, signatures of

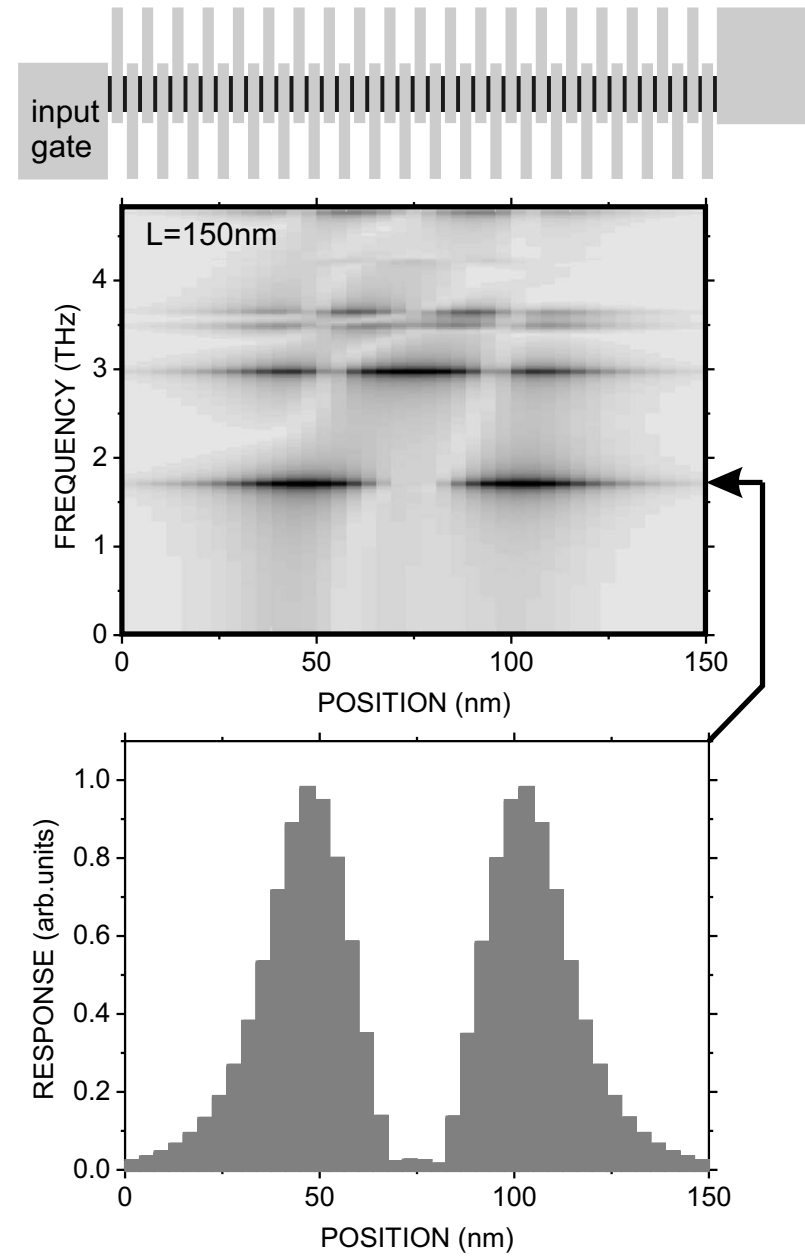

FIG. 5. Top view of the nanowire structure $(40+2$ gates $)$ and the simulated first-order $\mathrm{THz}$ transition spectrum for a GaAs nanowire with $L=150 \mathrm{~nm}$ and $d=20 \mathrm{~nm}$. The number of electrons is $N=4$. Within the grayscale plot, black corresponds to a strong response signal. For this device, the main effect of the electron-electron interaction consists mainly in a renormalization of the energy spectrum. The lower diagram shows an intensity cross section at the fundamental frequency indicated by the arrow.

the Wigner-like regime for the $600 \mathrm{~nm}$ case (Fig. 6) can be identified via the appearance of additional spatial peaks which are related to the oscillatory nature of the chargedensity wave. One has to note that the maxima in these transition spectra are located at those gate positions where the charge oscillates the most, and thus need not coincide with the charge-density maxima. In fact, for a noninteracting case, the spatial $\mathrm{THz}$ pattern is related to the gate-averaged product of the two wave functions of the involved final and destination states. Even with the inclusion of the electronelectron interaction, the considered $L=150 \mathrm{~nm}$ device comes close to such a situation. In the case of a correlated manybody state, however, such an interpretation is not applicable in general and a many-body approach such as the BBCI becomes mandatory. In any case, the obtained $\mathrm{THz}$ spectra can be considered as "fingerprints" of the concrete electronic configuration of the channel, providing not only information about electronic transition energies but also about the spatial configuration of the underlying few-electron states. 


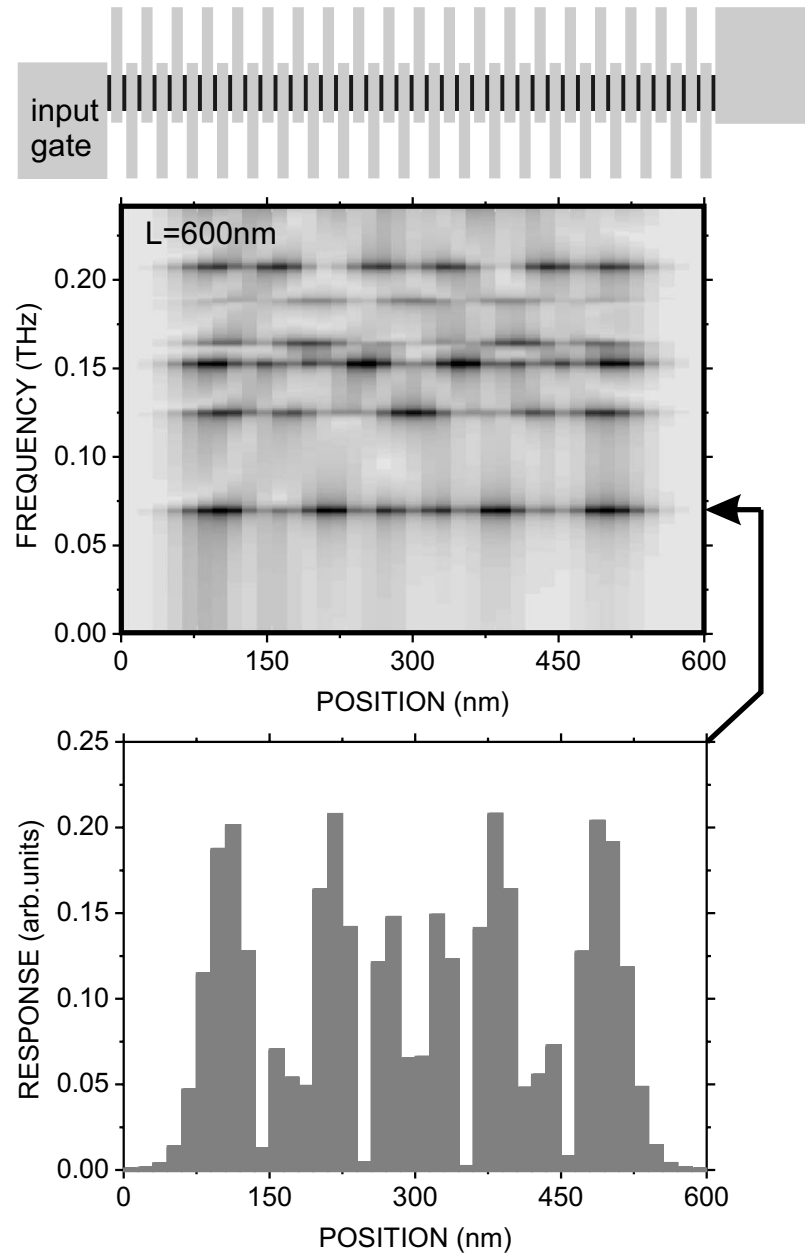

FIG. 6. Top view of the nanowire structure $(40+2$ gates $)$ and the simulated first-order $\mathrm{THz}$ transition spectrum for a GaAs nanowire with $L=600 \mathrm{~nm}$ and $d=20 \mathrm{~nm}$. The number of electrons is $N=4$. Within the grayscale plot, black corresponds to a strong response signal. The lower diagram shows an intensity cross section at the fundamental frequency indicated by the arrow. As compared to the short-channel case in Fig. 5, the formation of a Wigner molecule leads to additional spatial peaks.

\section{Reference calculation and numerical convergence}

In order to verify the convergence of the employed BBCI algorithm and to give an estimation of the energy error for the many-body spectrum, in this section we therefore provide a comparison of the BBCI results with a conventional full diagonalization (full CI) for a varying number of Focksubspace basis states. Figure 7 visualizes the simulation results for the lowest many-body eigenenergies of the nanowire system for two channel lengths $L=150 \mathrm{~nm}$ and $600 \mathrm{~nm}$. In both cases, $N=4$ electrons have been considered. The number of single-particle basis states is $N_{\max }=28$, resulting in a total number of $D_{\max }(4)=20475$ Slater determinants for the full-CI calculation. Here, the relative error

$$
\Delta_{n}=\frac{E_{n}^{\mathrm{BBCI}}-E_{n}^{\mathrm{FCI}}}{E_{n}^{\mathrm{FCI}}}
$$

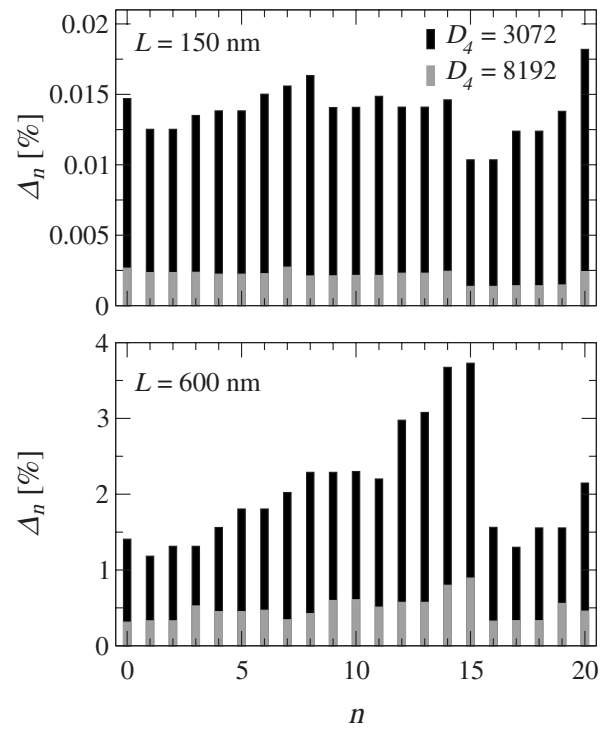

FIG. 7. Relative deviations of BBCI many-body eigenenergies compared to a full-CI reference calculation $\left[D_{\max }(4)=20475\right]$ as a function of the many-body eigenstate index $n$. The example shows two GaAs nanowire FETs with channel lengths $L=150$ and $600 \mathrm{~nm}$, respectively. In both cases, a diameter of $d=20 \mathrm{~nm}$ and a total number of $N=4$ electrons have been chosen. As a parameter, two Fock-subspace dimensions have been used for the BBCI: $D_{4}$ $=3072$ (black) and 8192 (gray).

is plotted as a function of the many-body eigenstate index $n$. $E^{\mathrm{BBCl}}$ and $E^{\mathrm{FCI}}$ denote the eigenenergies obtained from the BBCI and full-CI calculations, respectively. Two Focksubspace dimensions have been employed within the bucketbrigade selection scheme for the BBCI: $D_{4}=3072$ and 8192 . As can be seen, for the case of a short channel $L=150 \mathrm{~nm}$, the maximum error of the BBCI spectrum remains well below $0.02 \%$, even for 3072 BBCI Slater determinants only (as compared to 20475 for full CI). Considering the second case of the longer channel $L=600 \mathrm{~nm}$, one can clearly observe an increased energy error, which is due to the dominating Coulomb interaction in the Wigner regime. Nevertheless, for 8192 BBCI Slater determinants, the maximum error remains still below $1 \%$ for the first 20 excited many-body states. Obviously, a full-CI approach is numerically very time consuming or even impractical for some cases (such as $N_{\max }$ $=64$ single-particle states as employed in the previous sections). Here, the strength of the BBCI approach lies in a significantly reduced Fock-space dimension, requiring only a fraction of Slater determinants while providing results of a controllable accuracy. If we were solely interested in groundstate properties, computationally much more efficient methods exist, however, lacking excited many-body states which are provided by BBCI in a controlled manner.

The convergence of the BBCI selection process has also been tested by use of full-CI reference calculations. Figure 8 shows the calculated relative energy error as a function of the employed Fock-subspace dimension $D_{4}$. Apart from the ground state $(n=0)$, two representative many-body eigenstates $(n=9,19)$ are plotted as well. As can be clearly seen, the BBCI scheme converges for an increasing number of Slater determinants, finally approaching the full-CI result. 

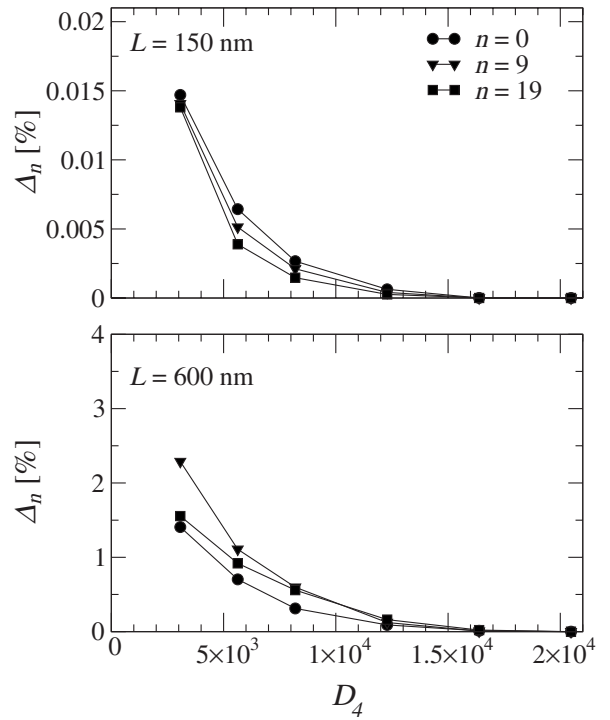

FIG. 8. Convergence test for the parameters as discussed in Fig. 7. The relative deviation of $\mathrm{BBCI}$ energies is plotted as a function of the Fock-subspace dimension $D_{4}$ for three representative eigenstates $(n=0,9,19)$.

The BBCI thus yields a controlled approximation, in particular for the numerical calculation of excited many-body states.

\section{SUMMARY}

We have discussed a recursive method to construct a subset of relevant Slater determinants for use in many-body diagonalization schemes in order to simulate many-body effects of realistic nanodevices with Coulomb interaction. The system is assumed to be finite. The described method becomes advantageous if a large number of single-particle basis states is required where full diagonalization schemes become impractical. By use of a recursive method, a reduced number (typically a few 1000) of relevant many-body basis states (Slater determinants) is generated, systematically scanning all given single-particle basis states. On this basis, a nonperturbative numerical approach to the calculation of the many-body statistical operator for an arbitrary periodic timedependent Hamiltonian has been formulated.

As a realistic example, we have considered a $\mathrm{THz}$ probe for a spatially resolved analysis of electronic spectra in nanowire-based transistors employing a multisegment gate design. We have simulated the $\mathrm{THz}$ response of few-electron quantum states in gated nanowire structures by use of our method. The discussed case of a GaAs-based device demonstrates that signatures of Wigner-like charge-density waves can be identified by use of this method, which lies beyond the scope of standard characterization methods based on stationary transport. As such, the proposed multigate $\mathrm{THz}$ probe technique might prove useful in a future experimental realization as a means to characterize nanoscale devices which are dominated by quantization and Coulomb effects.

\section{APPENDIX: BUCKET-BRIGADE ALGORITHM}

The bucket-brigade algorithm can be defined via the following recursion scheme as visualized in Fig. 1:

1. Start

"Empty buckets except for the vacuum"

For $J=0$ and $N=1, \ldots, N_{\max }: S_{J, N}=\varnothing$.

For $J=0, \ldots, N_{\max }$ and $N=0: S_{J, N}=\{|0\rangle\}$.

2. Recursion step $J \rightarrow J+1$ for $J=0, \ldots, N_{\text {max }}-1$

"Add single-particle state $J$ to existing Slater determinants and select"

For $N=1, \ldots, N_{\text {max }}$ :

(i) Expansion

Let $\hat{S}=S_{J, N} \cup\left\{c_{J}^{\dagger} v \mid v \in S_{J, N-1}\right\}$.

(ii) Truncation

If $\operatorname{card}(\hat{S}) \leq D_{N}$, choose $S_{J+1, N}=\hat{S}$

Else, choose $S_{J+1, N} \subset \hat{S}$ such that $\operatorname{card}\left(S_{J+1, N}\right)=D_{N}$ and $\forall v \in S_{J+1, N}, \forall w \in \hat{S} \backslash S_{J+1, N}: \mu(v) \leq \mu(w)$.

Here, $|0\rangle$ denotes the Fock space vacuum state, $\operatorname{card}(S)$ is the number of Slater determinants in set $S$, and $D_{N}$ (with $N$ $\left.=0, \ldots, N_{\max }\right)$ are given integers which determine the maximum number of states in $S_{J, N}$ (for all $J$ ). Hence, we must have $D_{N} \leq D_{\max }(N) \equiv\left(\begin{array}{c}N_{\max } \\ N\end{array}\right)$. Furthermore, $\mu$ is a real "measure of importance" for the selection of Slater determinants. For a normalized Slater determinant $v$, we assume a measure of the form $\mu(v)=\langle v|M| v\rangle$ with a many-body operator $M$ (which can be assumed to be diagonal without loss of generality). The latter can be expanded in a series of $n$-particle product terms

$$
M=\sum_{j_{1}} M_{j_{1}}^{(1)} N_{j_{1}}+\sum_{j_{1}<j_{2}} M_{j_{1} j_{2}}^{(2)} N_{j_{1}} N_{j_{2}}+\cdots,
$$

with $N_{j} \equiv c_{j}^{\dagger} c_{j}$ for single-particle state $j$ and real coefficients $M^{(n)}$, which represent conditional $n$-particle selection weights. In the simplest case (as in the example discussed in this paper), one can choose the diagonal elements of the Hamiltonian itself, that is,

$$
M_{j}^{(1)}=\epsilon_{j j}, \quad M_{j k}^{(2)}=V_{j k k j}-V_{j k j k}, \quad M^{(n>2)}=0 .
$$

This simple measure corresponds to a selection by energy in first-order perturbation theory. ${ }^{47}$ From this construction it is obvious that the set $S_{J, N}$ contains only Slater determinants with a total number of $N$ particles. To some extent, the described procedure resembles the NRG recursion scheme, ${ }^{32}$ however, lacking the costly diagonalization steps at this stage.

For any desired particle number $N_{0} \leq N_{\text {max }}$, we can now define a finite Fock subspace

$$
F_{0}=\operatorname{span}\left(S_{N_{\max }, N_{0}}\right),
$$

spanned by the many-body basis $S_{N_{\max }, N_{0}}$ of "relevant" Slater determinants with dimension 


$$
\operatorname{dim}\left(F_{0}\right)=\operatorname{card}\left(S_{N_{\max }, N_{0}}\right) \leq D_{N_{0}} .
$$

By construction, the states within $S_{N_{\max }, N_{0}}$ for a fixed $N_{0}$ are orthonormalized. In turn, a corresponding finite restricted Hamiltonian within $F_{0}$ can be diagonalized numerically in a matrix representation with respect to the basis $S_{N_{\text {max }}, N_{0}}$.

Within the BBCI algorithm, two means of (a posteriori) optimization exist. (i) Choice of the single-particle basis and in particular its order. (ii) Choice of the selection criterion $\mu$ of Slater determinants.

As for (i), one could solve an effective single-particle mean-field problem derived from $H_{0}$ for a given particle number $N_{0}$ and the given occupation conditions [e.g., Hartree-Fock, LDA-SDFT (Refs. 15-17)]. The resulting single-particle ON eigenbasis of this problem could be chosen for $B$. Furthermore, one can define an outer selfconsistency loop for the described algorithm. Here, the many-body state (preparation) of the system is described by a suitable many-body statistical operator $\rho\left[H_{0}\right]$ which is expressed in terms of the calculated basis $S_{N_{\max }, N_{0}}$ and the associated eigenvalues for a given boundary condition (such as maximum entropy under given constraints, nonequilibrium injection via rate equations, or the selection of a certain excited many-body state). In turn, the single-particle eigenstates of the resulting (transposed) single-particle density matrix $\hat{\rho}$, which can be calculated via

$$
\hat{\rho}_{k j}=\operatorname{Tr}\left(\rho c_{j}^{\dagger} c_{k}\right),
$$

are used as a new single-particle basis $B_{\text {new }}$ for a repeated many-body diagonalization, employing $S_{N_{\max }, N_{0}}\left[B_{\text {new }}\right]$. Such an approach resembles the MCSCF formalism ${ }^{23}$ (where these single-particle states are referred to as "natural orbitals"). The latter method typically aims at the calculation of one particular many-body state (e.g., the ground state). In any case, the correct choice of the single-particle basis is essential for any finite expansion of many-body states in Slater determinants to be efficient. ${ }^{48}$

As for (ii), the measure $\mu$ can also be chosen adaptively from the many-body diagonalization. Here, the coefficients $M^{(n)}$ might be constructed from the probability weights of conditional $n$-particle projection amplitudes of the calculated many-body statistical operator $\rho$. Implemented as a repeated selection+diagonalization (analogous to $\mathrm{MCCI}^{30,31}$ ) this defines a self-consistent optimization scheme for $\mu$. *m.indlekofer@fz-juelich.de

${ }^{1}$ D. K. Ferry and S. M. Goodnick, Transport in Nanostructures (Cambridge University Press, Cambridge, 1997).

${ }^{2}$ S. Datta, Electronic Transport in Mesoscopic Systems (Cambridge University Press, Cambridge, 1998).

${ }^{3}$ C. Thelander, T. Martensson, M. T. Björk, B. J. Ohlsson, M. W. Larsson, L. R. Wallenberg, and L. Samuelson, Appl. Phys. Lett. 83, 2052 (2003).

${ }^{4}$ M. Suzuki, K. Ishibashi, K. Toratani, D. Tsuya, and Y. Aoyagi, Appl. Phys. Lett. 81, 2273 (2002).

${ }^{5}$ P. Jarillo-Herrero, S. Sapmaz, C. Dekker, L. P. Kouwenhoven, and H. S. J. van der Zant, Nature (London) 429, 389 (2004).

${ }^{6}$ J. Wensorra, K. M. Indlekofer, M. Lepsa, A. Förster, and H. Lüth, Nano Lett. 5, 2470 (2005).

${ }^{7}$ J. Guo, S. Datta, and M. Lundstrom, IEEE Trans. Electron Devices 51, 172 (2004).

${ }^{8}$ K. M. Indlekofer, J. Knoch, and J. Appenzeller, Phys. Rev. B 72, 125308 (2005)

${ }^{9}$ R. Lake, G. Klimeck, R. C. Bowen, and D. Jovanovic, J. Appl. Phys. 81, 7845 (1997).

${ }^{10}$ D. L. John and D. L. Pulfrey, Phys. Status Solidi B 243, 442 (2006).

${ }^{11}$ P. W. Anderson, Phys. Rev. 124, 41 (1961).

12 J. Hubbard, Proc. R. Soc. London, Ser. A 276, 238 (1963).

${ }^{13}$ K. M. Indlekofer, J. Knoch, and J. Appenzeller, Phys. Rev. B 74, 113310 (2006).

${ }^{14}$ K. M. Indlekofer, J. Knoch, and J. Appenzeller, IEEE Trans. Electron Devices 54, 1502 (2007).

${ }^{15}$ For a review see R. Dreizler and E. Gross, Density Functional Theory (Plenum Press, New York, 1995), and references therein.

${ }^{16}$ W. Kohn and L. J. Sham, Phys. Rev. 140, A1133 (1965).

${ }^{17}$ J. P. Perdew and A. Zunger, Phys. Rev. B 23, 5048 (1981).
${ }^{18}$ W. M. C. Foulkes, L. Mitas, R. J. Needs, and G. Rajagopal, Rev. Mod. Phys. 73, 33 (2001).

${ }^{19}$ D. M. Ceperley, Rev. Mod. Phys. 67, 279 (1995).

${ }^{20}$ A. V. Filinov, Yu. E. Lozovik, and M. Bonitz, Phys. Status Solidi B 221, 231 (2000).

${ }^{21}$ S. M. Reimann and M. Manninen, Rev. Mod. Phys. 74, 1283 (2002).

${ }^{22}$ K. M. Indlekofer and H. Lüth, Phys. Rev. B 62, 13016 (2000).

${ }^{23}$ A. Szabo and N. S. Ostlund, Modern Quantum Chemistry: Introduction to Advanced Electronic Structure Theory (Dover, Mineola, NY, 1996).

${ }^{24} \mathrm{~F}$. Jensen, Introduction to Computational Chemistry (Wiley, Chichester, 2006).

${ }^{25}$ Th. Müller, in Computational Condensed Matter Physics, 37th Spring School of the Institute of Solid State Research, Jülich (Research Centre Jülich GmbH, Jülich, Germany, 2006).

${ }^{26}$ R. Dronskowski, Computational Chemistry of Solid State Materials (Wiley-VCH, Weinheim, 2005).

${ }^{27}$ C. D. Sherrill and H. F. Schaefer, Adv. Quantum Chem. 34, 143 (1999).

${ }^{28}$ P. G. Szalay, M. Nooijen, and R. J. Bartlett, J. Chem. Phys. 103, 281 (1995)

${ }^{29}$ Y. G. Khait, A. Azenkeng, H. Wang, T. J. Dudley, and M. R. Hoffmann, J. Chem. Phys. 122, 094111 (2005).

${ }^{30}$ J. C. Greer, J. Comput. Phys. 146, 181 (1998).

${ }^{31}$ W. Győrffy, Th. M. Henderson, and J. C. Greer, J. Chem. Phys. 125, 054104 (2006).

${ }^{32}$ K. G. Wilson, Rev. Mod. Phys. 47, 773 (1975).

${ }^{33}$ S. R. White, Phys. Rev. Lett. 69, 2863 (1992).

${ }^{34}$ J. H. Shirley, Phys. Rev. 138, B979 (1965).

${ }^{35}$ K. F. Milfeld and R. E. Wyatt, Phys. Rev. A 27, 72 (1983).

${ }^{36}$ R. Kubo, J. Phys. Soc. Jpn. 12, 570 (1957). 
${ }^{37}$ P. W. Langhoff, S. T. Epstein, and M. Karplus, Rev. Mod. Phys. 44, 602 (1972).

${ }^{38}$ J. H. Eberly and K. Wódkiewicz, J. Opt. Soc. Am. 67, 1252 (1977).

${ }^{39}$ L. P. Kouwenhoven, S. Jauhar, J. Orenstein, P. L. McEuen, Y. Nagamune, J. Motohisa, and H. Sakaki, Phys. Rev. Lett. 73, 3443 (1994).

${ }^{40}$ S. Kasai, W. Han, M. Yumoto, and H. Hasegawa, Phys. Status Solidi C 0, 1329 (2003).

${ }^{41}$ E. Räsänen, H. Saarikoski, V. N. Stavrou, A. Harju, M. J. Puska, and R. M. Nieminen, Phys. Rev. B 67, 235307 (2003).

${ }^{42}$ The usage of our method to calculate higher order, i.e., nonlinear response spectra will be published elsewhere.

${ }^{43}$ C. P. Auth and J. D. Plummer, IEEE Electron Device Lett. 18, 74 (1997).
${ }^{44}$ C. W. J. Beenakker, Phys. Rev. B 44, 1646 (1991).

${ }^{45}$ W. Häusler and B. Kramer, Phys. Rev. B 47, 16353 (1993).

${ }^{46}$ Assuming an oscillating image charge of $0.1-1$ electrons at 1 $\mathrm{THz}$, we could expect an ac current on the order of $10^{-8}-10^{-7} \mathrm{~A}$ per nanowire. In our case, the load of a typical microstrip line of $50 \Omega$ could still be considered as a short circuit with negligible feedback on the device.

${ }^{47}$ In the context of ground-state CI, a selection based on secondorder perturbation theory has been employed by C. F. Bender and E. R. Davidson, Phys. Rev. 183, 23 (1969).

${ }^{48}$ In fact, even a single-Slater-determinant state might require a large CI expansion in an unsuitable single-particle basis. Here, the "single-particle entropy" $-k_{B} \operatorname{Tr}(\hat{\rho} \ln \hat{\rho})$ could be used as a measure of true correlation within a given pure many-body state. 